\title{
APPLIANCE
}

\section{PERSPEX PROSTHESIS FOR THE LACRIMAL SAC AND NASO-LACRIMAL DUCT*}

\author{
BY
}

\author{
L. H. G. MOORE \\ Dudley, Worcestershire
}

THIS technique has been used as a simple alternative to dacryocystorhinostomy. The results in a small series of cases are sufficiently encouraging to suggest that it might be worth a trial in a wider field.

\section{Construction}

The prosthesis is made of "Transpex I" (I.C.I.). The top part is $12 \mathrm{~mm}$. long, $5 \mathrm{~mm}$. in diameter, and has a bore of $3 \mathrm{~mm}$. It is perforated like a colander with sixteen holes $1.75 \mathrm{~mm}$. in diameter. The lower part is $15 \mathrm{~mm}$. long, $3 \mathrm{~mm}$. in diameter, and has a bore of $1.5 \mathrm{~mm}$. The top part is at an angle of $20^{\circ}$ to the lower part.

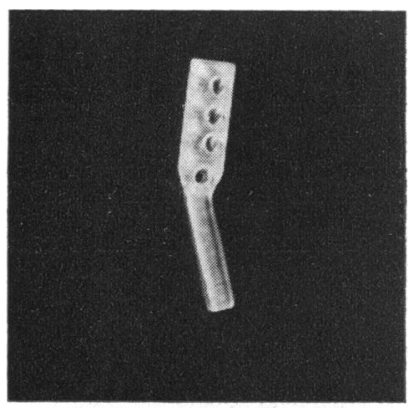

FIGURE.-Photograph of prosthesisactual size.

\section{Sterilization}

The following method was adopted after other methods, including heat, had failed. The prosthesis was washed in distilled water. It was then placed in a 20-ml. polythene weighing bottle (Gallenkamp). A sterile 10 per cent caustic soda solution was poured into the bottle, the polythene top was replaced, and it was then placed in a water bath at $30^{\circ} \mathrm{C}$. for one hour. The 10 per cent. solution was then decanted and replaced by a 0.1 per cent. sterile solution of caustic soda. The polythene cap was replaced, and the weighing bottle complete with its contents was placed in a sterile glass bottle with a screw top, and was stored in this way until required at operation. Immediately before use the prosthesis was washed in sterile normal saline.

\section{Insertion}

General anaesthesia (Flaxedil, Thiopentone, Halothane, gas and oxygen, and headtilt) was used in all of the cases. The lower punctum was dilated. A No. 1 Bowman's

* Received for publication May 7, 1958. 
probe was passed down the sac and if possible into the duct. This was felt through the skin, and using a Bard-Parker knife (No. 15 blade) an incision was made $2 \mathrm{~mm}$. below the punctum through all layers to expose the probe. Blunt scissors were spread in a vertical direction on both sides of the probe. The incision was $15 \mathrm{~mm}$. in length. The field was kept free from discharge and haemorrhage by the use of a sucker. The probe was then withdrawn and a No. 8 Bowman's probe was passed through the wound and down the naso-lacrimal duct, using force if necessary to do this. As this probe was withdrawn, the lower part of the prosthesis was passed into the duct, and the upper part was rotated into the sac. The wound was dusted with penicillin and sulphathiazole powder. No sutures were used. The operation was performed in from 4 to 5 minutes.

\section{Post-Operative Care}

A firm dressing and pressure bandage were applied, and after 24 hours this was removed and a collodion gauze dressing was applied. This was removed on the first visit to the out-patient clinic, 6 days after operation. The wound healed by first intention in all but one of the cases.

Penicillin was given by intra-muscular injection twice daily, 250,000 units for 2 days. The patient was then discharged from hospital, and was given oral penicillin V tablets $240 \mathrm{mg}$. 1 four times a day for 4 days, and penicillin eye drops 50,000 units $/ \mathrm{ml}$. to use thrice daily for 1 or 2 weeks. The patient was advised to avoid sneezing and nose-blowing for 10 days, and 9 days after the operation the tear passages were syringed.

\section{Case Reports}

Case 1. Mucocoele of left sac was present for 3 years with severe bronchitis. A prototype prosthesis was inserted in September, 1957. This one was straight and formed a swelling almost as large as the original mucocoele. It was tender to touch and so in December, 1957, the straight prosthesis was removed and an angled one inserted. The patient was symptom-free in April, 1958, and fluid passed easily into the nose on syringing.

Case 2. Epiphora present on the left side for $3 \frac{1}{2}$ years was not relieved by repeated syringing and probing. A prosthesis was inserted in October, 1957, with immediate relief of symptoms. In March, 1958, the patient had a cold and afterwards developed epiphora again. He was given hyalase drops to use for one week, and the obstruction which was present was then relieved by syringing through the lower punctum while applying pressure with chalazion forceps to the upper punctum and canaliculus. He reported free from symptoms one month later.

Case 3. Epiphora present on left side for 2 years was not relieved by syringing and probing. A prosthesis was inserted on the left side in December, 1957, and she became symptom free and fluid passed into the nose on syringing during the next 6 weeks. She had remained free from symptoms when last seen at the end of March, 1958.

Case 4. Epiphora and regurgitation of muco-pus from the right sac had been present for 2 years. A prosthesis was inserted on the right side in December, 1957, and the patient was symptom free until April, 1958, when she complained of slight watering of the right eye. Fluid passed easily into the nose on syringing and she again became free from symptoms. 
Case 5. Both eyes had watered for 3 months, with tenderness over the right sac and conjunctivitis on the right side. Both tear passages were obstructed and were not relieved by syringing. After four visits to the out-patient clinic it was decided to insert a prosthesis on each side. This was done in November, 1957. Both tear passages were patent on syringing in December, 1957, and again in January, 1958. Unfortunately this patient died suddenly while at work a few weeks later, and the cause of death at autopsy was found to be cardiac infarct due to coronary artery disease.

Case 6. Seen in June, 1957, with an attack of acute dacryocystitis on the left side, which was stated to be the sixth attack within a few months. This settled down with treatment but the eye remained watery. At the end of November an acute attack of dacryocystitis occurred again, and it was decided to put in a prosthesis while the condition was in an acute phase. This was done but was not successful. The wound did not heal perfectly at its upper limit and, although fluid could be passed easily into the nose by syringing either through the punctum or through the fistula, the inflammation did not subside. The prosthesis was removed in January, 1958, no attempt being then made at further surgery. In April, 1958, there was a mucocoele of the sac with epiphora, but no inflammation and no fistula. It is hoped to persuade the patient to have another prosthesis inserted within a short time.

Case 7. A mucocoele of the right sac present for 6 months was not relieved by outpatient measures and a prosthesis was inserted in April, 1958. The patient became symptom-free and stated that he could see better at work with the right eye. This was probably because he had been getting a regurgitation of glairy mucus into the conjunctival sac before operation.

\section{Conclusion}

It would seem that this technique yields a high proportion of successes in cases of simple epiphora and of mucocoele.

The advantages are these:

(i) The operation takes only a few minutes to perform.

(ii) The patient is kept in hospital for only 3 days.

(iii) In the event of failure, further surgical procedures should not be difficult.

\section{Summary}

A technique is described for insertion of a perspex prosthesis which will intubate the naso-lacrimal duct and fill the potential space cavity of the lacrimal sac. It is suggested that this may prove a simple alternative to dacryocystorhinostomy.

My thanks are due to Dr. R. M. Archer, who assisted at most of the operations and removed the prosthesis from Case 6 in my absence, and to Dr. J. P. Murphy who gave the anaesthetics for these cases ; to Dr. Gordon and his laboratory staff for confirmatory work with B. subtilis spores; to Mr. F. Byron, the pharmacist, for his help with the sterilization experiments ; and to the sisters and nurses at the Guest Hospital, Dudley, who have taken such an interest in these cases.

Last and by no means least, I am most grateful to Mr. P. M. Caudell of Messrs. Rayner's instrument department, who bore with patience my many changes of mind before the prosthesis reached its final shape and size. 